\title{
Das Leitlinien-Clearingverfahren COPD. Empfehlungen für eine nationale Leitlinie
}

\author{
D. Franzen ${ }^{1}$ \\ J. Fessler ${ }^{2}$ \\ J. Fischer ${ }^{3}$ \\ M. Geraedts ${ }^{4}$ \\ H. J. Graf ${ }^{5}$ \\ H. U. Kauczor 6 \\ C. Kroegel ${ }^{7}$ \\ K. Mörike ${ }^{8}$ \\ I. Kopp ${ }^{9}$ \\ D. Sonntag ${ }^{10}$
}

\author{
The Guideline Clearing Project COPD. Recommendations for a National Guideline
}

\section{Zusammenfassung}

Zur Qualitätsförderung der Gesundheitsversorgung von Patienten mit COPD verabredeten die Spitzenverbände der Selbstverwaltungskörperschaften im Gesundheitswesen die Durchführung eines Clearingverfahrens zu Leitlinien für die Behandlung von Patienten mit COPD. Nach systematischer Literaturrecherche wurden die Empfehlungen von 20 aktuellen deutsch- bzw. englischsprachigen Leitlinien mit überregionaler Gültigkeit formal anhand der Checkliste „Methodische Qualität von Leitlinien“ bewertet. Ein multidisziplinär zusammengesetzter Expertenkreis der Clearingstelle des Ärztlichen Zentrums für Qualität in der Medizin (ÄZQ) überprüfte die Inhalte der Leitlinienempfehlungen im Hinblick auf Angemessenheit und Praktikabilität im deutschen Gesundheitssystem. Bei der Bewertung der methodischen Qualität konnten die meisten Punkte für die Behandlungsleitlinie zur COPD der Veterans Health Administration (US) vergeben werden, gefolgt von der der Deutschen Atemwegsliga und der Deutschen Gesellschaft für Pneumologie. Für eine Weiterentwicklung der nationalen Leitlinie in Deutschland wurden Vorschläge zu 19 inhaltlichen Eckpunkten erarbeitet, deren Berücksichtigung im Hinblick auf Struktur und Inhalt empfohlen werden. Danach beruht eine angemessene Versorgung von Patienten mit COPD unter anderem auf der Entwicklung einer an

\section{Abstract}

In order to promote the care of patients with COPD in Germany a national guideline clearing project was initiated jointly by autonomous corporate bodies of the German health care system. Following a systematic search of literature data bases between 1992 and 2002, 20 guidelines were identified that met the inclusion criteria and were evaluated with the German Checklist for Methodological Guideline Appraisal. Following this, a multidisciplinary expert group appointed by the German Guideline Clearinghouse (Leitlinien-Clearingstelle im Ärztlichen Zentrum für Qualität in der Medizin, ÄZQ) reviewed the suitability of these guidelines for the use in the German health care system. Referring to methodological aspects, criteria were best met by the guideline of the Veteran's Health Administration/Department of Defense (US), followed by the one of the Deutsche Atemwegsliga and the Deutsche Gesellschaft für Pneumologie. Aiming at the production respectivly a revision of a German national guideline for COPD the expert group agreed on recommendations organized in 19 chapters. Among others these strengthened the role of a precise definition of COPD based primarily on the pathogenesis, of a subtle description of all diagnostic and therapeutic tools and of a detailed description of quality assurance and quality management. The feasability of recommendations were demonstrat-

Institutsangaben

${ }^{1}$ Praxis für Herz- und Lungenkrankheiten, Köln

2Praxis für Allgemeinmedizin, Flörsheim

${ }^{3}$ Klinik Norderney mit Schwerpunkt Pneumologie/ LVA Westfalen

${ }^{4}$ Public Health, Heinrich-Heine-Universität Düsseldorf

${ }^{5}$ Praxis für Lungenkrankheiten, Köln

${ }^{6}$ Deutsches Krebsforschungszentrum Heidelberg

${ }^{7}$ Pneumologie \& Allergologie, Klinik für Innere Medizin I, Friedrich-Schiller-Universität Jena

${ }^{8}$ Abteilung Klinische Pharmakologie, Institut für Pharmakologie und Toxikologie,

Universitätsklinikum Tübingen

${ }^{9}$ Arbeitsgemeinschaft der Wissenschaftlichen Medizinischen Fachgesellschaften (AWMF),

Philipps-Universität Marburg

${ }^{10}$ Ärztliches Zentrum für Qualität in der Medizin, Köln

Korrespondenzadresse

PD Dr. med. Damian Franzen·Berrenratherstr. 296 · 50937 Köln · E-mail: franzen.damian@t-online.de

Bibliografie

Pneumologie 2004; 58: 858-862 @ Georg Thieme Verlag KG Stuttgart · New York

DOI 10.1055/s-2004-830135

ISSN 0934-8387 
der Pathogenese orientierten Definition der Erkrankung, einer subtilen Darstellung und Bewertung aller einzusetzenden diagnostischen und therapeutischen Maßnahmen sowie der Darstellung geeigneter Verfahren zur Qualitätssicherung und wirkungsvoller Instrumente zur Implementierung der Leitlinie. Anhand ausgewählter Beispiele wurden die Empfehlungen für die Erstellung bzw. Aktualisierung einer nationalen Leitlinie konkretisiert. Darüber hinaus bietet sich die Nutzung der Ergebnisse dieses Verfahrens zu Steuerungszwecken im Gesundheitswesen an. ed by examples chosen from the evaluated guidelines. Additionally the presented findings may be used as steering tools in the German Health care system.

\section{Einführung}

Zur Qualitätsförderung der Gesundheitsversorgung von Menschen mit COPD wurde ein Leitlinien-Clearingverfahren zur vergleichenden Qualitätsdarlegung von Methodik und Inhalt ärztlicher Therapieleitlinien durchgeführt [1]. In dem Leitlinien-Clearingverfahren kooperieren Bundesärztekammer, Kassenärztliche Bundesvereinigung, Deutsche Krankenhausgesellschaft, die Spitzenverbände der gesetzlichen und privaten Krankenversicherungen und die Gesetzliche Rentenversicherung. Eines der wesentlichen Ziele dieses Projekts ist die Erarbeitung von Empfehlungen, die bei der Entwicklung einer nationalen Leitlinie beachtet werden sollen.

\section{Methodik}

Analog zu anderen, bereits abgeschlossenen Leitlinien-Clearingverfahren [2], wurde mittels systematischer Literaturrecherche nach deutsch- und englischsprachigen Leitlinien zur Behandlung der COPD, aber auch der „chronischen Bronchitis“ und von „akuten Exazerbationen" gesucht. Dabei konnten insgesamt 538 Literaturangaben zu ärztlichen Therapieleitlinien, die zwischen 1992 und 2002 erschienen sind, identifiziert werden. Nach Ausschluss von Dubletten bzw. Literaturzitaten wurden 48 Leitlinien im Volltext gesichtet. Unter der Berücksichtigung der überregionalen Gültigkeit dieser Leitlinien, sowie des Versorgungsbereiches „Rehabilitation“ der COPD wurden letztlich 20 Leitlinien in das Clearingverfahren eingeschlossen (Tab.1).

Die methodischen Aspekte der eingeschlossenen Leitlinien wurden anhand der Checkliste „Methodische Qualität von Leitlinien“ (2. Version, 8/1999) [3] bewertet. Diese ist in drei Bereiche geordnet: 1. die zur Leitlinienentwicklung herangezogenen Verfahren, 2. die Qualität der Darstellung und der Formate der Leitlinienempfehlungen und 3. denjenigen Faktoren, die mit der Realisierung der Empfehlungen verbunden sind. Im ersten und zweiten Bereich der Checkliste werden Fragen zu 17 verschiedenen Sachverhalten gestellt und sechs weitere im dritten Bereich, die die Aspekte der Implementierung und Anwendbarkeit erfassen. Durch Summation der möglichen „Ja“-Antworten ist damit eine Gesamtpunktzahl von maximal 40 Punkten erreichbar.

Ein vom Steuergremium des Clearingverfahrens multidisziplinär zusammengesetzter Expertenkreis führte die Bewertung der Inhalte der aufgenommenen Leitlinien durch. Beteiligt waren Vertreter der Allgemeinmedizin, der Inneren Medizin, der Pneumo-
Tab. 1 Im Leitlinien-Clearingverfahren COPD bewertete Leitlinien

LL01 Alberta Medical Association. Guideline for the management of acute exacerbations of chronic bronchitis (AECB)/acute and chronic/COPD. 2001. p. 1 - 7. Available from: URL: http://www.albertadoctors.org/ resources/cpg/guidelines/aecb.pdf

LL02 American Academy of Family Physicians. Chronic bronchitis: primary care management. Am Fam Physicians 1998; 57: 2365-2372, 2376-2378. Available from: URL: http://www.aafp.org/afp/ 980515ap/heath.html

LL03 American Board of Family Practice. COPD. 2001. p. 1-59. Available from: URL: http://www.familypractice.com/references/guidesframe.htm

LL04 American College of Chest Physicians, American College of Chest Physicians/American Association of Cardiovascular and Pulmonary Rehabilitation. Pulmonary rehabilitation. Chest 1997; 112: $1363-1396$. Available from: URL: $=$ Apr\&fyear $=1965 \&$ tmonth $=$ Aug\&tyear $=2002 \&$ hits $=10$ \&journalcode $=$ chest $\&$ fdatedef $=1+$ April $+1965 \&$ tdatedef $=1+$ August +2002

LL05 American College of Physicians, American Society of Internal Medicine, American College of Chest Physicians. Part I: Evidence base for the management of acute exacerbations of chronic obstructive pulmonary disease. Ann Intern Med 2001; 134: 595 - 599. Part II: Management of acute exacerbations of chronic obstructive pulmonary disease: a summary and appraisal of published evidence. Ann Intern Med 2001; 134: 600-620. Available from: URL: Part I: http://www.annals.org/issues/v134n7/pdf/200104030-00015.pdf, Part II: http://www.annals.org/issues/v134n7/pdf/ 200104030-00016.pdf

LL06 American Thoracic Society. Standards for the diagnosis and care of patients with chronic obstructive pulmonary disease. Am J Respir Crit Care Med 1995; 152: S77-120. Available from: URL: www.thoracic.org/adobe/statements/copd1 - 45.pdf

LL07 British Thoracic Society. Guidelines on the management of COPD. Thorax 1997; 52 Suppl. 5: S1-32

LL08 British Thoracic Society. Rehabilitation. Thorax 2001; 56: 827-834

LL09 Canadian Respiratory Review Panel, Department of Family and Community Medicine, University of Toronto. Guidelines for the treatment of chronic obstructive pulmonary disease (COPD). Toronto: Oti/Mums; 1998

LL10 Deutsche Atemwegsliga, Deutsche Gesellschaft für Pneumologie. Leitlinie der Deutschen Atemwegsliga und der Deutschen Gesellschaft für Pneumologie zur Diagnostik und Therapie von Patienten mit chronisch obstruktiver Bronchitis und Lungenemphysem (COPD). Pneumologie 2002; 56: 704 - 738. Available from: URL: http://www.atemwegsliga.de/copd/index.htm

LL11 European Respiratory Society. Optimal assessment and management of chronic obstructive pulmonary disease (COPD). Eur Respir J 1995; 8: 1398 - 1420. Available from: URL: http://www.ersnet.org/0/0/0.asp

LL12 Finnish Medical Society Duodecim. Chronic obstructive pulmonary disease (COPD). 2002. p.1-6. Available from: URL: http://www.ebm-guidelines.com

LL13 Institute for Clinical Systems Improvement. Chronic obstructive pulmonary disease. 2003. p.1-62. Available from: URL: http://www.icsi.org 


\section{Tab. 1 Fortsetzung}

LL14 Laitinen LA, Koskela K. Chronic bronchitis and chronic obstructive pulmonary disease: Finnish national guidelines for prevention and treatment 1998-2007. Respiratory Medicine 1999; 93: 297-332

LL15 Ministry of Health of Malaysia, Academy of Medicine of Malaysia, Malaysian Thoracic Society. Guidelines in the management of chronic obstructive pulmonary disease. Medical Journal of Malaysia 1999; 54: 387-401

LL16 New Zealand Guidelines Group, The COPD Board NRGDG. Guidelines to best practice for management of stable chronic obstructive pulmonary disease (COPD). 1999. p. 1-34. Available from: URL: http://www.nzgg.org.nz/library/gl_complete/copd/index.cfm

LL17 South Africa Medical Association. Guidelines for the management of chronic obstructive pulmonary disease. SAM] 1998; 88: 999-1010

LL18 Swiss Respiratory Society. Management of chronic obstructive pulmonary disease: the Swiss guideline. Swiss Medical Weekly 2002; 132: 67 - 78. Available from: URL: http://www.smw.ch/ set_archiv.html

LL19 Veterans Health Administration, Department of Defense. VHA/DOD clinical practice guideline for the management of chronic obstructive pulmonary disease. 1999. p. 1-116. Available from: URL: http://www.oqp.med.va.gov/cpg/COPD/COPD_base.htm

LL20 World Health Organization and National Heart, Lung and Blood Institute. Global strategy for the diagnosis, management, and prevention of chronic obstructive pulmonary disease. 2003. Available from: URL: http://www.goldcopd.com/

logie, der Kardiologie, der Radiologie, der Rehabilitationsmedizin, der Klinischen Pharmakologie und des Public Health. Die verschiedenen Versorgungsbereiche des bundesdeutschen Gesundheitssystems wurden bei der Besetzung explizit berücksichtigt. Der Expertenkreis wurde durch eine auf dem Gebiet der Entwicklung von Leitlinien und der evidenzbasierten Medizin erfahrenen Ärztin moderiert.

Bei der inhaltlichen Beurteilung wurden zum einen die Empfehlungen der Leitlinien zu vergleichbaren klinischen Fragestellungen untereinander verglichen. Zum anderen überprüfte der Expertenkreis, in welchem Ausmaß die Empfehlungen im deutschen Gesundheitssystem umsetzbar sind und inwieweit sie dessen Belange abdecken. Auf dieser Analyse aufbauend, formulierten die Experten Vorschläge, die bei der Erstellung einer künftigen nationalen Leitlinie zur Behandlung von Patienten mit COPD berücksichtigt werden sollten. Anhand einer Auswahl vorbildlicher Beispiele aus den bewerteten Leitlinien in Form von Textstellen, Tabellen oder Grafiken wurde die Umsetzbarkeit der Vorschläge dargelegt.

\section{Ergebnisse der methodischen Bewertung}

Anhand der Bewertung mittels der Checkliste „Methodische Qualität von Leitlinien“ erzielte die Leitlinie des amerikanischen Verteidigungsministeriums mit 27 von 40 (erreichbaren) Punkten den höchsten Wert (Tab. 1). Diese Punktsumme ist vergleichbar mit den besten Bewertungsergebnissen aus Clearingverfahren zu anderen Versorgungsproblemen (z.B. aus den LeitlinienClearingverfahren „Diabetes mellitus Typ 1“ oder „Depression“). Die Qualität der Verfahren der Leitlinienentwicklung wurde durchschnittlich mit 5 von 17 Punkten niedriger bewertet als die Qualität der Darstellung von Inhalten und Format. Für diesen methodischen Bereich konnten durchschnittlich 10 von 17 Punkten vergeben werden. Bei der Beurteilung von Aspekten der Implementierung und Evaluation wurde meist ein Punkt für Verfügbarkeit von z.B. Online-, Kurz- und Langversionen oder Patientenversionen vergeben. Nur zwei Leitlinien halten Angaben vor, wie der Einsatz der LL überprüft werden kann (z.B. Qualitätsindikatoren). Im Durchschnitt konnte hier weniger als ein Punkt vergeben werden. Die Leitlinie der Deutschen Atemwegsliga und der Deutschen Gesellschaft für Pneumologie erfüllt die Kriterien einer evidenzbasierten Leitlinie [4] und enthält eine anhand des DISCERN-Instrumentes [5] hochwertige Patientenversion [6]. Mit insgesamt 24 Punkten wird sie im Vergleich der methodischen Qualität der Leitlinien in diesem Clearingverfahren an zweiter Stelle geführt.

\section{Ergebnisse der Beurteilung der Inhalte}

Die bewerteten Leitlinien enthalten überwiegend systematisch abgehandelte und teilweise äußerst umfangreiche Darstellungen. Im Vergleich fallen Unterschiede hinsichtlich der Strukturierung und der inhaltlichen Schwerpunktsetzung. auf. Viele Empfehlungen der Leitlinien sind oft nur eingeschränkt im deutschen Versorgungssystem umsetzbar, bzw. werden dessen Belange nicht ausreichend davon abgedeckt. Der Expertenkreis entwickelte aufbauend auf der Problemanalyse einen Anforderungskatalog zu insgesamt 19 inhaltlichen Eckpunkten, die von einer nationalen Leitlinie berücksichtigt werden sollen.

In der Einzelbeurteilung entsprach zwar keine der in das Clearingverfahren eingeschlossenen Leitlinien vollständig den geforderten Vorstellungen. Es konnte jedoch eine umfangreiche Anzahl von Einzelempfehlungen aus der Gesamtzahl der bewerteten Leitlinien auswählen, die den Erfordernissen des deutschen Gesundheitssystems gerecht werden. Im Folgenden werden relevante Aspekte der inhaltlichen Bewertung der analysierten Leitlinien dargestellt.

Der Definition der COPD wurde eine zentrale Rolle bei der Erstellung einer künftigen Leitlinie beigemessen. Je nach Zielsetzung und/oder Sichtweise der Verfasser waren die Darstellungen der Definition der COPD in den erfassten Leitlinien sehr unterschiedlich. Insbesondere unter Berücksichtigung präventiver Aspekte ist jedoch nach Ansicht der Experten eine an der Pathogenese orientierte Definition der COPD dringend erforderlich. Diese sollte auch die für die COPD besonders wichtigen Prodromalphasen (z. B. die chronische Bronchitis) und Risikokonstellationen (inhalativer Tabakkonsum, etc.) einschließen. Häufig wird in den Leitlinien außerdem nicht berücksichtigt, dass die klinischen Manifestationen der COPD in Ausprägung und Progression großen individuellen Schwankungen unterworfen und durch mehr oder minder häufige Exazerbationen gekennzeichnet sind. Neben der Darstellung der teilweise erheblichen physischen Beeinträchtigungen ist die COPD auch mit sozialen und emotionalen Folgen belastet. Wie deren Einschätzung erfolgen soll, wird in den Leitlinien meist nicht angegeben.

Nach Vorstellungen des Expertenkreises erfordert die Diagnostik der COPD klare, sich an den Inhalten der Definition orientierende Kriterien, die eine Einordnung des Krankheitsbildes durch die 
Bestimmung von Ausprägung, Schweregrad und Prognose ermöglichen. Neben einer subtilen Beschreibung und Bewertung der einzelnen objektiven Meßmethoden erfordern sowohl die subjektiven als auch die klinischen Symptome gleichermaßen eine entsprechende Berücksichtung. Diese Ausführungen sind jeweils für die Positiv- bzw. Erst-, Ausschluss-, Exazerbationsoder Komplikationsdiagnostik in separaten Abhandlungen zu besprechen. In der Beschreibung des Stellenwertes verschiedener diagnostischer Verfahren, ihrer Praktikabilität und ihres Aufwandes liegen die Schlüsselelemente eines adäquaten und rationalen Vorgehens. Den Perspektiven neuartiger, z.B. molekulargenetischer, genetischer, mitarbeitsunabhängiger, nicht-invasiver oder strahlungsfreier diagnostischer Verfahren, für die teilweise noch erheblicher Forschungsbedarf besteht, wurde in den vorhandenen Leitlinien meist nicht ausreichend Rechnung getragen.

Die Therapieziele sollen auf der Grundlage einer präzisen Beschreibung der Erkrankung, ihren verschiedenen Manifestationen, ihrer Ausprägung und der Schweregrade entwickelt werden. Dabei soll ein Weg aufgezeigt werden, wie zwischen den von den Behandlern beabsichtigten Zielen und den Präferenzen der Patienten vermittelt werden kann, da die fehlende Abstimmung des Therapiekonzeptes eine häufig unterschätzte Ursache nicht realisierbarer Therapieziele darstellt.

Durch Präventionsmaßnahmen wie Tabakabstinenz, Impfungen und Berufswahl bzw. Substitutionstherapien bei Mangelsyndromen kann der Ausbruch der COPD gemildert, verzögert und z.T. auch verhindert werden. Hier wurde die chronische Bronchitis als relevante Vorstufe zur COPD in den vielen Leitlinien nicht als Kriterium für einzuleitende präventive Maßnahmen benannt. Obwohl fast alle Leitlinien der Prävention ein Kapitel gewidmet haben, waren Verfahren zur Nikotinentwöhnung, und insbesondere solche, die in Laienkreisen sehr große Verbreitung finden, gar nicht oder nur in wenig hilfreicher Form erwähnt. Hinweise auf sportliche Betätigung und Schulungsmaßnahmen wurden nicht durchgängig als Bestandteil der Prävention genannt.

In den Leitlinien werden die Indikationskriterien für die Rehabilitation nur ungenügend aufgeführt. Es erfolgt auch meist keine ausreichende Abgrenzung der Rehabilitation von Kuration und Prävention. Die Benennung von Inhalten und Zielen der verschiedenen Formen der Rehabilitation und die Zuordnung von Zuständigkeiten für deren Festlegung fehlt in den meisten Leitlinien. Wünschenswert erscheint daher u.a. eine inhaltliche Ausgestaltung von Schnittstellen für den Zugang zur und die Nachsorge nach der Rehabilitation. Die rehabilitativen Therapieziele sollten im somatischen, psychosozialen und edukativen Bereich individuell mit dem Patienten beraten und festgelegt werden.

Eine zukünftige nationale Leitlinie zur Behandlung von Patienten mit COPD erfordert neben den statistischen Angaben zur Wirksamkeit medikamentöser und nicht-medikamentöser Therapien eine differenzierte Wertung von Nutzen und Risiken, die Nebenwirkungen, Kontraindikationen und relevante Wechselwirkungen berücksichtigen soll. Auftretende therapeutische Konflikte bei der Behandlung von Begleiterkrankungen wie auch wenig validierte oder obsolete Therapien sollten gesondert benannt werden. Nur sehr wenige Leitlinien erwähnen häufig angewandte al- ternative Therapieverfahren (z. B. Homöopathie oder Naturheilverfahren); diese sollten jedoch auch hinsichtlich ihrer Wirksamkeit und Sicherheit bewertet werden. Die chirurgischen Maßnahmen nehmen nur einen geringen Raum bei den zu berücksichtigenden Therapieoptionen ein, bedürfen jedoch ebenfalls einer kritischen Wertung hinsichtlich ihrer Therapieziele, Indikationen und Kontraindikationen.

Jede eingesetzte Therapie bedarf einer adäquaten Nachsorge und kontinuierlichen Betreuung. Infolge des individuellen Krankheitsverlaufes, mangelnder Compliance oder nicht erreichter Therapieziele (z. B. frustraner Versuch der Nikotinentwöhnung), sind immer wieder Korrekturen von Therapieplänen und Zielen erforderlich. In meisten Leitlinien fehlten solche Kontrollsysteme, die dem Patienten eine richtige Einschätzung seiner Erkrankung und dem Arzt eine angemessene Behandlungsführung und Beurteilung der Lebensqualität des Patienten erlauben.

Die Probleme der Versorgungskoordination werden durchgängig in nicht ausreichendem Maße angesprochen. Die notwendigen Grundlagen zur Verbesserung des Qualitätsmanagements bei der Behandlung der COPD fehlen in den bewerteten Leitlinien meist. Dabei könnten dadurch z.B. Versorgungslücken aufgedeckt oder Verbesserungsvorschläge erarbeitet werden. Der Güte der wirtschaftlichen Versorgung sollte in Leitlinien mehr Aufmerksamkeit zukommen.

Die Leitlinien enthalten nur in unzureichendem Ausmaß Implementierungs-Strategien. Dazu würden z.B. Hinweise auf Analysen zur Erkennung von möglichen Barrieren, die die Nutzung der Leitlinie behindern, gehören. Von den bewerteten Leitlinien sind solche, die begleitend Patienten- oder auch Kurzversionen (z. B. im Kitteltaschenformat) vorhalten, in der Minderzahl. Als wichtigster Vorschlag zu diesem Eckpunkt, soll eine zukünftige Leitlinie zur Behandlung der COPD auf verschiedenen Ebenen multimodale Maßnahmen der Implementierung benennen, damit ihre Empfehlungen wirkungsvoll umgesetzt werden können. Darüber hinaus bedarf es der Benennung von konkreten Maßnahmen zur Überprüfung und Rückkopplung hinsichtlich der Effektivität von Leitlinien.

\section{Schlussfolgerungen}

Im Leitlinien-Clearingverfahren „COPD“ hat ein Expertenkreis unter Geschäftsführung der Clearingstelle des ÄZQ aktuelle Therapieleitlinien zur COPD hinsichtlich Methodik und Inhalt bewertet. Im Konsens wurden in einem unabhängigen Expertenkreis Vorschläge für eine nationale Leitlinie zu diesem Versorgungsproblem erarbeitet. Fragen zur Definition des Krankheitsbildes, der Stellenwert diagnostischer und therapeutischer Verfahren, Kontrollsysteme und die Aspekte der Implementierung von Leitlinien spielten eine führende Rolle. Neben der Forderung, die methodische Qualität von Leitlinien zu verbessern, beinhaltet der abschließende Clearingbericht einen umfassenden Katalog von Vorschlägen, die der Verbesserung der Güte der medizinischen Versorgung und der Wirtschaftlichkeit bei der Behandlung von Patienten mit COPD dienen. 
Dieser Leitlinien-Clearingbericht ist auch als Empfehlung für das methodische und inhaltliche Vorgehen bei der Überarbeitung bestehender, bzw. Entwicklung zukünftiger Leitlinien zur COPD zu verstehen. Potenzielle Nutzer erhalten damit die Möglichkeit, vollständige Leitlinien oder auch nur Einzelempfehlungen zu identifizieren, die für die Lösung individueller Fragestellungen geeignet erscheinen. Mit Hilfe des Clearingberichtes können auf regionaler wie auch auf nationaler Ebene die Neu- bzw. Weiterentwicklung einer Leitlinie oder entsprechende lokale Anpassungsprozesse initiiert werden. Er dient somit als Instrument zur Vermeidung bzw. Behebung von Fehlern und Mängeln der Erstellung oder Überarbeitung von Leitlinien. Dieses LeitlinienClearingverfahren zeigt aber auch auf, wie die Erstellung bzw. die Aktualisierung einer nationalen Leitlinie auf bereits bestehende Leitlinien abgestützt werden könnte. Die in Kooperation zwischen der Deutschen Atemwegsliga und der Deutschen Gesellschaft für Pneumologie entstandene Leitlinie „COPD“ stellt mit ihrer international hochrangigen methodischen Qualität eine gute Ausgangsbasis dafür dar.

Für Steuerungszwecke entwickeln die kooperierenden Partner im Clearingverfahren einen so genannten Maßnahmenkatalog. Dieser enthält u.a. an die Gremien der Selbstverwaltung bzw. an die Fachgesellschaften und die Berufverbände gerichtete Empfehlungen zur Umsetzung der Ergebnisse des LeitlinienClearingberichtes.
Die Ergebnisse dieses Verfahrens sind im Leitlinien-Clearingbericht COPD zusammengestellt, der im Internet unter www.leitlinien.de eingesehen werden kann, bzw. als Printmedium in der Schriftenreihe des ÄZQ veröffentlicht wurde (zu bestellen im Fachbuchhandel oder unter www.medicombooks.de).

\section{Literatur}

${ }^{1}$ Ollenschläger G, Thomeczek C, Bungart B et al. Das Leitlinien ClearingProgramm der Selbstverwaltungskörperschaften im Gesundheitswesen - Ein Projekt zur Qualitätsförderung in der Medizin. Gesundheitswesen 1999; 61: $105-111$

2 Thole H, Weingart O, Lampert U et al. Das Leitlinien-Clearingverfahren Asthma bronchiale - 1. Methodik und Ergebnisse der formalen Bewertung. Pneumologie 2003; 57: 459-467

3 Ärztliche Zentralstelle Qualitätssicherung. Checkliste „Methodische Qualität von Leitlinien“. Dtsch Arztebl 1998; 95: A-2576-2578, C-1838-1840. Verfügbar unter: http://www.leitlinien.de

${ }^{4}$ Helou A, Lorenz W, Ollenschläger G et al. Methodische Standards der Entwicklungen evidenz-basierter Leitlinien in Deutschland. Z Arztl Fortbild Qualitätssich 2000; 94: 330-339

${ }^{5}$ Ärztliche Zentralstelle Qualitätssicherung. Das DISCERN-Handbuch. München: Zuckschwerdt, 2000. Verfügbar unter: http://www.discern.de/instrument.htm

${ }^{6}$ Deutsche Atemwegsliga. Behandlung von chronischer Bronchitis und Lungenemphysem. Informationen für Patienten. Verfügbar unter: http://www.atemwegsliga.de/download/info_chronbron_lungenemphysem.pdf. 